\title{
Estudo sobre a influência da espessura e glandularidade da mama na dose glandular média em exames de tomossíntese
}

Study on the influence of breast thickness and breast glandularity on the mean glandular dose in tomosynthesis exams

\author{
C. Engler ${ }^{1 *}$; M. R. P. Attie ${ }^{2}$; L. Paixão ${ }^{3}$; M. S. Nogueira ${ }^{1}$ \\ ${ }^{1}$ Laboratório de Radioproteção Aplicada à Mamografia, Centro de Desenvolvimento da Tecnologia Nuclear \\ (CDTN), 31270-901, Belo Horizonte - MG, Brasil \\ ${ }^{2}$ Departamento de Física, Universidade Federal de Sergipe (UFS), 49100-000, São Cristóvão - SE, Brasil \\ ${ }^{3}$ Departamento de Anatomia e Imagem, Faculdade de Medicina da Universidade Federal de Minas \\ Gerais (UFMG), 30130-100, Belo Horizonte - MG, Brasil \\ *camila.engler@cdtn.br \\ (Recebido em 26 de setembro de 2019; aceito em 18 de dezembro de 2019)
}

\begin{abstract}
A dose glandular média (DGM) é a grandeza dosimétrica utilizada para estimar a dose de radiação à qual uma paciente é exposta ao realizar um exame de mamografia. Este estudo tem como objetivo verificar a relação entre espessura da mama comprimida (EMC) e a densidade volumétrica da mama (DVM) com a DGM, assim como a relação da EMC e idade da paciente com a DVM. As imagens de tomossíntese mamária de 660 pacientes foram analisadas usando o software Volpara. A partir dessa análise, obteve-se a DVM e a DGM. A idade do paciente e EMC foram obtidas do cabeçalho DICOM das imagens. Utilizando o software estatístico SPSS, foram realizados testes de correlação entre a EMC e DVM com a DGM e entre a EMC e idade da paciente com a DVM. Para as correlações entre DVM e DGM, a grandeza EMC foi fixada de $10 \mathrm{em} 10 \mathrm{~mm}$. As correlações foram analisadas através dos coeficientes de correlação (r). Na relação entre DGM e EMC foi obtido $r=0,696$. Para a relação DGM e DVM, a melhor correlação foi encontrada na faixa de $60<\mathrm{EMC}<70 \mathrm{com} r=0,616$. As correlações entre idade da paciente e EMC com DVM apresentaram $r=-0,432$ e $r=-0,338$, respectivamente. Os resultados mostram que a EMC é o fator mais relacionado ao aumento da DGM enquanto que a DVM é ligeiramente mais afetada pela idade da paciente do que pela EMC.

Palavras-chaves: Mamografia, Dose Glandular Média, Densidade Volumétrica da Mama.
\end{abstract}

Mean glandular dose (MGD) is the dosimetric quantity used to estimate the radiation dose to which a patient is exposed during a mammogram examination. This study aims to verify the relationship between compressed breast thickness (CBT) and breast volumetric density (BVD) with MGD, as well as the relationship of CBT and patient's age with BVD. Breast tomosynthesis images of 660 patients were analyzed using the Volpara software. From this analysis, the BVD and MGD were obtained. Patient age and CBT were taken from the DICOM header of the images. Using the SPSS statistical software, correlation tests were performed between the CBT and the BVD with the MGD and between the CBT and the patient's age with the BVD. For correlations between BVD and MGD, the CBT was set at $10 \mathrm{~mm}$ thickness ranges. The correlations were analyzed through the correlation coefficients ( $r$ ). In the relationship between MGD and CBT, $r=0.696$ was found. For a MGD and BVD relationship, the best correlation was found in the range of $60<\mathrm{CBT}<70$ with $\mathrm{r}=0.616$. Correlations between patient age and CBT with BVD were $r=-$ 0.432 and $\mathrm{r}=-0.338$, respectively. The results show that CBT is the most related factor to the MGD increase, while the BVD is more affected by the patient's age than by the CBT.

Keywords: Mammography, Mean Glandular Dose, Breast Volumetric Density.

\section{INTRODUÇÃO}

A mamografia é a técnica de diagnóstico e rastreamento mais aceita para deteç̧ão precoce de neoplasias mamárias. A taxa de mortalidade precoce de mulheres na faixa etária de 40 a 69 anos, como consequência do câncer de mama, tem reduzido devido ao uso desse exame para rastreamento [1]. A dose de radiação que um paciente recebe quando realiza um exame mamográfico é um dos fatores mais importantes a ser considerado na implementação de uma nova tecnologia de rastreamento [2]. Ainda que o risco de câncer induzido por radiação é pequeno em 
relação ao benefício obtido com os exames de rastreamento, o medo causado pela radiação é um motivo que leva muitas mulheres a não realizarem o exame mamográfico de rastreamento [3].

A tomossíntese digital de mama é uma nova tecnologia de mamografia de rastreamento. Esse exame permite adquirir uma série de imagens da mama em várias posições angulares conforme $\mathrm{o}$ tubo de raios $\mathrm{X}$ se move em forma de arco e o feixe de radiação atravessa um trajeto definido pela mama. Posteriormente, as múltiplas imagens são reconstruídas por meio de algoritmo computacional, fornecendo uma imagem tridimensional. Cada projeção é realizada com uma dose relativamente pequena e a dose global do exame é comparável com a mamografia digital [4]. Os exames de tomossíntese apresentam resultados satisfatórios em relação à diminuição da sobreposição dos tecidos da mama e estão sendo indicados principalmente em casos nos quais as mamas são extremamente densas [5].

Para estimar a dose de radiação que um paciente recebe ao realizar um exame de mamografia ou tomossíntese é utilizada a grandeza dose glandular média (DGM). Esta grandeza é definida como a dose média absorvida no tecido glandular de uma mama comprimida em um exame de mamografia [6]. A estimativa dessa grandeza é feita pela medição do kerma no ar incidente multiplicado por coeficientes de conversão que dependem da glandularidade e da espessura da mama bem como características do espectro de raios $\mathrm{X}$ utilizado [7, 8].

A DGM absorvida por um paciente em um exame mamográfico de rastreamento pode variar devido a aspectos intrínsecos do paciente, dentre esses, os mais citados na literatura são a espessura da mama comprimida (EMC) e a densidade da mama. A EMC é vista em vários estudos como o fator que mais influência no valor da DGM [9-11]. Já a correlação da densidade da mama com a DGM é encontrada na literatura com valores mais baixos [12,11], com uma correlação inversa com a DGM [12] ou com falta de correlação com a DGM [11]. A densidade da mama é determinada pela quantidade de tecido glandular presente na mesma. Uma mama pode variar de completamente gordurosa até extremamente densa [13]. Essa variação pode estar associada a raça, aumento da idade e até mesmo ao uso de medicamentos [14].

Existem vários métodos utilizados para determinar a densidade mamária. Dentre eles, o Volpara é um programa computacional que utiliza imagens brutas (sem processamento) no formato DICOM para calcular a densidade volumétrica da mama (DVM). Para isto, o software determina o volume de tecido glandular pela integração da espessura do tecido denso em cada pixel da imagem. Já o volume total da mama é calculado pela multiplicação da área da mama com a espessura. Finalmente, a DVM é obtida pela razão entre o volume denso e o volume total da mama. Posteriormente, o software utiliza a DVM específica de cada paciente para cálculo da DGM de maneira individualizada [15].

O objetivo desse estudo é verificar como se comportam os fatores DVM e EMC na contribuição para a DGM em exames de tomossíntese em uma amostra de mulheres brasileiras. Além disso, serão verificadas fontes de influência para a mudança da DVM, uma vez que ela é um fator que pode apresentar variação ao longo da vida de uma mulher.

\section{MATERIAL E MÉTODOS}

A coleta de imagens deste estudo foi submetida à apreciação para aprovação do Comitê de Ética da Faculdade de Ciências Médicas de Minas Gerais de acordo com o protocolo CAAE: 18934019.2.0000.5134. O consentimento informado do paciente não foi obtido, uma vez que este trabalho foi realizado de maneira retrospectiva.

A amostra de imagens é constituída de exames de tomossíntese de rastreamento nas projeções craniocaudal (CC) e médio-lateral obliqua (MLO), com EMC entre 30 e $100 \mathrm{~mm}$, realizados entre os anos de 2016 e 2017. Foram coletadas imagens clínicas de pacientes das regiões Centro-Oeste (209 pacientes), Nordeste (33 pacientes) e Norte (418 pacientes) do Brasil, totalizando 660 pacientes. Em todos os equipamentos cujas imagens foram coletadas, foi verificada a realização dos testes de controle de qualidade e assegurada a conformidade dos mesmos. Na Tabela 1 constam informações técnicas dos aparelhos de tomossíntese de mama de cada região. 
Tabela 1: Informações técnicas dos aparelhos de tomossíntese de mama.

\begin{tabular}{cccc}
\hline Região & $\begin{array}{c}\text { Aparelho } \\
\text { mamográfico }\end{array}$ & Ânodo/Filtro & $\begin{array}{c}\text { Número de } \\
\text { cortes por projeção }\end{array}$ \\
\hline \multirow{2}{*}{ Centro Oeste } & Ge Essential ADS & $\mathrm{Rh} / \mathrm{Rh}$ & 9 cortes \\
& GE Pristina & $\mathrm{Rh} / \mathrm{Ag}$ ou & 9 cortes \\
\multirow{2}{*}{ Norte } & GE Pristina & $\mathrm{Mo} / \mathrm{Mo} \mathrm{ou}$ & 9 cortes \\
\multirow{2}{*}{ Nordeste } & GE Essential ADS & $\mathrm{Rh} / \mathrm{Ag}$ & 9 cortes \\
& Hologic Selenia & $\mathrm{Wh} / \mathrm{Al}$ & 8 cortes \\
\hline
\end{tabular}

O software Volpara (Volpara Solutions, Versão 1.1, Wellington, New Zelândia) foi utilizado para realizar a análise das imagens. Como resultado, o Volpara fornece uma planilha eletrônica com diversas informações sobre a composição da mama, DGM e aspectos relacionados ao exame mamográfico. Da análise das imagens clínicas com o Volpara, foram selecionadas as grandezas DVM e DGM. A idade das pacientes e a EMC foram retiradas do cabeçalho DICOM das imagens.

O Volpara, se baseia na metodologia Dance para o cálculo da DGM, utilizando a seguinte equação:

$$
\operatorname{MGD}(\mathrm{mGy})=\mathrm{K} \mathrm{g} \mathrm{c} \mathrm{s}
$$

No entanto, o Volpara só utiliza o (k), Kerma incidente no ar, e os fatores de conversão (g) e (s), que converte o kerma incidente no ar em $D G M$ para uma mama padrão e corrige o efeito de combinações anodo/filtro diferentes de Mo/Mo, respectivamente. O fator de conversão (c), responsável por corrigir para porcentagens de tecido adiposo e glandular diferente de 50\%/50\% não é utilizado, visto que o software usa a densidade específica de cada paciente no lugar de (c) [15].

Cada paciente dispunha de quatro imagens, sendo uma de cada projeção (CC e MLO) para cada uma das mamas. Para a determinação dos parâmetros avaliados para cada paciente, foi realizada a média das informações obtidas das quatro imagens. Todos os dados foram coletados e organizados em uma planilha eletrônica de dados.

O software Statistical Package for the Social Sciences (SPSS) foi utilizado para análises estatísticas [16]. Os testes Kolmogorov-Smirnov e Shapiro-Wilk foram utilizados para verificar se as grandezas seguiam uma distribuição gaussiana, $p>0,05$. As grandezas que seguiram uma distribuição gaussiana foram representadas pela média, enquanto que as grandezas que não seguiram essa distribuição foram representadas pela mediana. Em seguida os testes Pearson e Spearman foram empregados para analisar a correlação bivariável entre as grandezas. O teste de Pearson foi utilizado quando as duas grandezas apresentavam distribuição gaussiana enquanto que o teste Spearman foi utilizado quando uma das grandezas ou ambas não apresentavam distribuição gaussiana. Os testes de correlação foram realizados entre a DGM e EMC, DGM e DVM, fixando a grandeza EMC em intervalos de $10 \mathrm{~mm}$ de espessura, DVM com EMC e DVM com idade da paciente. Os resultados das correlações bivariáveis foram analisados através do coeficiente de correlação (r) e aceitos com nível de confiança estatístico de $95 \%(p<0,05)$.

\section{RESULTADOS E DISCUSSÃO}

A amostra de pacientes apresentou uma média da variável EMC de $59( \pm 12) \mathrm{mm}$ e as demais variáveis apresentaram medianas de 51 anos de idade (intervalo de 25 a 87 anos), DGM de 1,53 mGy (intervalo de 0,43 a 4,68 mGy) e DVM de 7,78\% (intervalo de 2,16\% a 36,40 \%).

Nos testes para verificação da distribuição da curva, apenas as grandezas nas quais os dois testes, Kolmogorov-Smirnov ou Shapiro-Wilk, resultaram em um valor de $p>0,05$ foram tratadas como distribuição gaussiana. Os resultados dos testes de verificação de distribuição estão dispostos na Tabela 2. 
Tabela 2: Resultados dos testes de verificação da distribuição das variáveis.

\begin{tabular}{cc}
\hline Variável & Valor de $\boldsymbol{p}$ \\
\hline DGM & $p<0,05$ \\
DVM & $p<0,05$ \\
EMC & $p>0,05$ \\
Idade da paciente & $p<0,05$ \\
DGM $(30<$ EMC $<40)$ & $p<0,05$ \\
DGM $(40<$ EMC $<50)$ & $p<0,05$ \\
DGM $(50<$ EMC $<60)$ & $p<0,05$ \\
DGM $(60<$ EMC $<70)$ & $p<0,05$ \\
DGM $(70<$ EMC $<80)$ & $p<0,05$ \\
DGM $(80<$ EMC $<100)$ & $p<0,05$ \\
DVM $(30<$ EMC $<40)$ & $p<0,05$ \\
DVM $(40<$ EMC<50) & $p<0,05$ \\
DVM $(50<$ EMC $<60)$ & $p>0,05$ \\
DVM $(60<$ EMC $<70)$ & $p<0,05$ \\
DVM $(70<$ EMC $<80)$ & $p<0,05$ \\
DVM $(80<$ EMC $<100)$ & $p<0,05$ \\
\hline
\end{tabular}

No teste de correlação entre DGM e EMC, resultou em um r =0,696 $(p<0,05)$. Na Figura 1 é possível visualizar que o acréscimo da DGM é condizente com o aumento da EMC. Mamas com maior EMC atenuarão mais intensamente a radiação com a necessidade de uso de um feixe de raios $\mathrm{X}$ mais energético do que nos casos onde a EMC é menor. Esses resultados concordam com outros trabalhos, nos quais os autores também encontraram a melhor correlação positiva entre DGM e EMC entre os fatores estudados $[11,12]$.

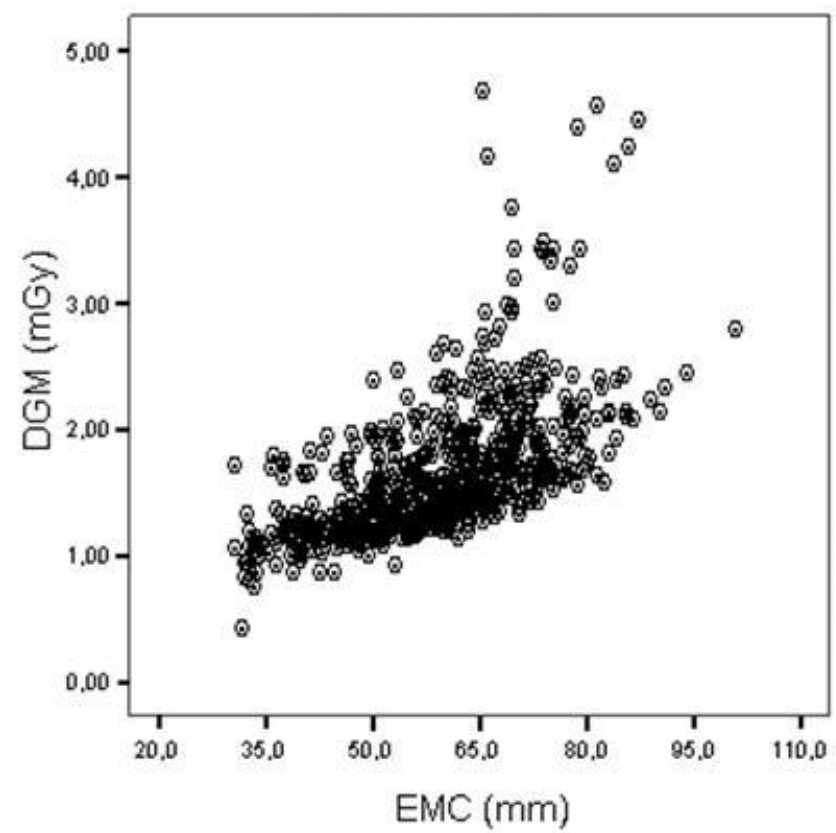

Figura 1: DGM em função da EMC.

A variável DVM também apresentou uma correlação positiva com a DGM. Os coeficientes de correlação foram calculados fixando a grandeza EMC de $10 \mathrm{em} 10 \mathrm{~mm}$ para uma análise mais aprimorada. A melhor correlação foi encontrada para o subgrupo de $60<\mathrm{EMC}<70, \mathrm{r}=0,616$ ( $p$ $<0,05)$, seguida de $70<\mathrm{EMC}<80, \mathrm{r}=0,578(p<0,05), 40<\mathrm{EMC}<50 \mathrm{r}=0,483(p<0,05), 30$ $<\mathrm{EMC}<40, \mathrm{r}=0,479,(p<0,05), 50<\mathrm{EMC}<60, \mathrm{r}=0,372(p<0,05)$ e $80<\mathrm{EMC}<100, \mathrm{r}=$ $0,321(p<0,05)$. O tecido glandular atenua mais os raios $\mathrm{X}$ que o tecido adiposo. Portanto, uma 
mama com alta densidade absorverá uma proporção maior da radiação do que uma mama mais adiposa e da mesma espessura [17].

Este resultado é semelhante ao encontrado por Nguyen et al. (2016) [11] e de Shin et al. (2016) [12]. Estes pesquisadores também encontraram, entre a DVM e a DGM, a menor correlação entre os fatores analisados. Em outro estudo foi encontrada uma correlação inversa entre a DGM e a densidade da mama [18]. Uma alta densidade da mama aumenta a atenuação da radiação e, por consequência, resulta em uma maior DGM [19], entretanto, no presente estudo, a baixa densidade volumétrica mamária $(7,78 \%)$ da amostra de pacientes analisada contribui para que esse efeito na DGM seja menor, principalmente quando comparado à influência da EMC.

Na Figura 2 pode ser visto que nos três subgrupos de EMC em que a DGM aumentou gradualmente com o aumento da DVM e todas as faixas de DVM estavam presentes ( $40<$ EMC $<50,60<\mathrm{EMC}<70$ e $70<\mathrm{EMC}<80$ e) foram encontradas as melhores correlações entre DVM e DGM.

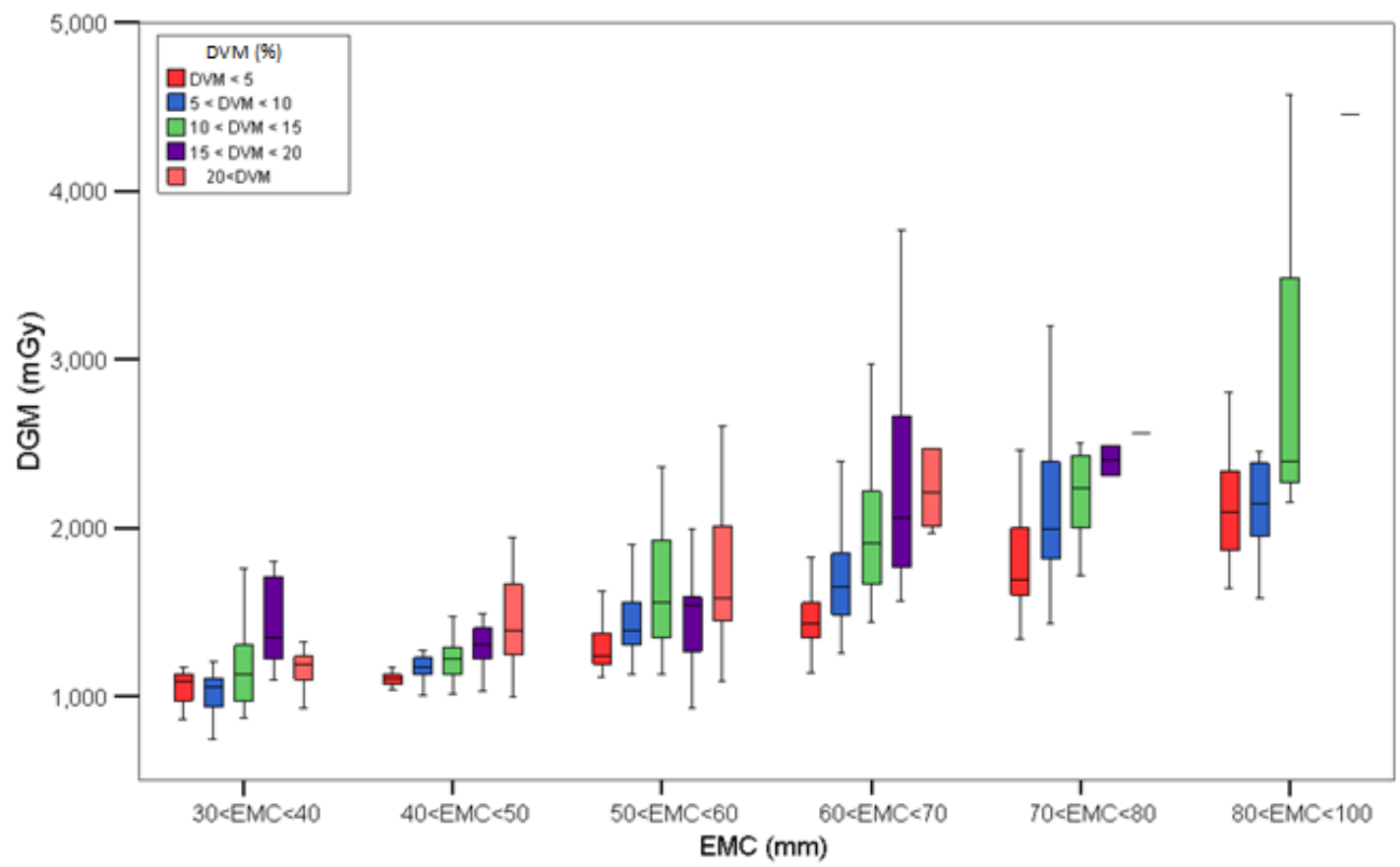

Figura 2: DGM em função da EMC e DVM.

Devido ao fato de que a glandularidade da mama é um fator que sofre variabilidade em diferentes tipos de mama e em diferentes idades das mulheres, foram realizadas análises de correlação entre a DVM e a EMC e entre a DVM e a idade da paciente. Ambas se correlacionaram negativamente com a DVM. A maior correlação encontrada foi entre a DVM e a idade da paciente, com $\mathrm{r}=-0,432(p<0,05)$, enquanto que a DVM e EMC resultou em uma correlação $\mathrm{r}=-0,338$ $(p<0,05)$. Na Figura 3 é possível visualizar uma tendência de diminuição da DVM em mulheres mais velhas. Portanto, quanto maior a idade da paciente menor a quantidade de tecido denso na mama. Na Figura 4, verifica-se que em mamas de maior EMC a DVM está propensa a diminuir. 


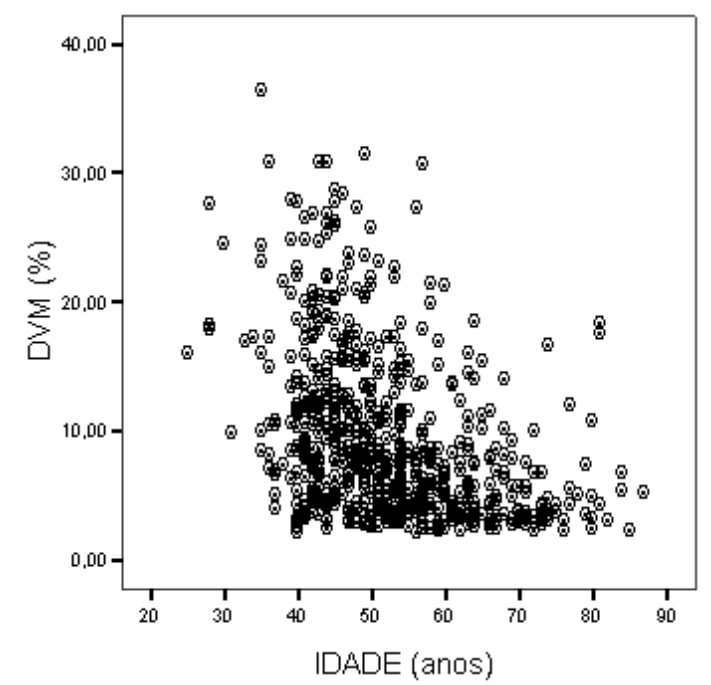

Figura 3: DVM em função da Idade da paciente.

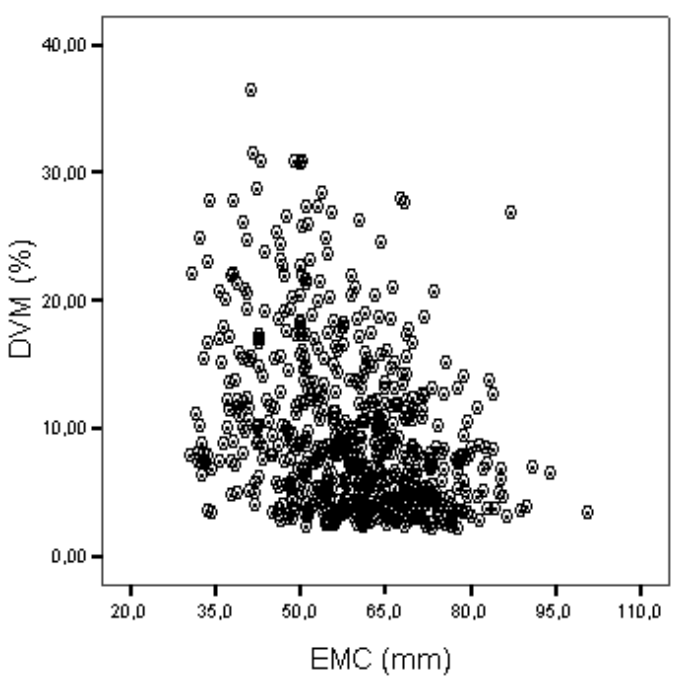

Figura 4: DVM em função EMC.

Neste estudo, os coeficientes de correlação para a idade da paciente com a DVM e EMC com a DVM resultaram em valores baixos, sendo assim não se deve concluir nada somente a partir destes. Entretanto, foram realizadas comparações com outros estudos realizados que apresentaram resultados similares a estes.

Os resultados referentes à relação da DVM com a idade da paciente são semelhante ao de Zulfiqar et al. (2011) [14], no qual a quantidade de tecido glandular na mama sofreu um declínio com o aumento da idade. Em outro estudo foi constatado que a densidade da mama é afetada pela idade da paciente e, ainda, que em mulheres mais jovens a acurácia da mamografia é menor devido à alta densidade da mama [20]. Esses resultados são coerentes, já que estudos mostram que com o aumento da idade o tecido glandular da mama é substituído por tecido adiposo, deixando-a mais flácida. Essas alterações nas estruturas mamárias podem começar já aos 30 anos, quando os níveis do hormônio folículo-estimulante começam a aumentar progressivamente até a menopausa [14].

Resultados similares aos deste trabalho foram encontrados por outros autores referentes à relação da DVM com a EMC [21, 22]. Essa correlação inversa ocorre pelo fato de que em mamas de maior volume, existe uma menor proporção de tecido glandular. O volume da mama é diretamente associado à EMC [23].

A relevância deste trabalho foi mostrar o efeito da DVM e da EMC na DGM assim como o comportamento da DVM, em relação a variação de fatores influentes, utilizando informações de uma amostra de mulheres brasileiras. Ainda que resultados como este sejam conhecidos em outros países, estudos com amostras variadas de pacientes são importantes, visto que as características da mama, como EMC e DVM são fatores intrínsecos da mama e, portanto, distintos em mulheres de diferentes países.

Algumas limitações são encontradas neste estudo. Não foram consideradas as características intrínsecas de cada modelo de equipamento mamográfico, como a combinação anodo/filtro. Também não foi avaliada a influência das variáveis associadas ao espectro do feixe do raio $\mathrm{X}$ $(\mathrm{kV}, \mathrm{mAs})$. Visto que esses fatores contribuem no cálculo da DGM, na metodologia Dance,[24], na qual o software Volpara se baseia para o cálculo da DGM, caso tivessem sido considerados, resultados com maior embasamento poderiam ter sido encontrados.

\section{CONCLUSÃO}

Nesse trabalho, a EMC mostrou ter maior contribuição para o aumento da dose de radiação em um exame de mamografia. A interferência da DVM na dose glandular surgiu somente quando analisada com a grandeza EMC fixada e, ainda assim, inferior à influência da EMC. A DVM apresentou uma maior variação com a idade da paciente quando comparado com a EMC.

Portanto, uma compressão adequada da mama e uma informação singular da densidade de cada paciente podem contribuir para uma estimativa mais assertiva da DGM. 


\section{AGRADECIMENTOS}

Os autores agradecem ao CNPq (Projeto $n^{\circ}$ 401331/2014-8), FAPEMIG (Projeto ${ }^{\circ}$ PPM00643-16) e CNEN pelo apoio financeiro e as instituições que colaboraram com as imagens utilizadas nesse estudo.

\section{REFERÊNCIAS BIBLIOGRÁFICAS}

1. Gartlehner G, Thaler K, Chapman A, Kaminski-Hartenthaler A, Berzaczy D, Van Noord MG, et al. Mammography in combination with breast ultrasonography versus mammography for breast cancer screening in women at average risk. Cochrane Database Syst Rev. 2013 Apr; 30(4):CD009632, doi: 10.1002/14651858.CD009632.

2. Di Maria S, Baptista M, Felix M, Oliveira N, Matela N, Janeiro L, et al. Optimal photon energy comparison between digital breast tomosynthesis and mammography: A case study. Phys Medica. 2014 Feb;30(4):482-8, doi: 10.1016/j.ejmp.2014.02.001.

3. Aro AR, De Koning HJ, Absetz P, Schreck M. Two distinct groups of non-attenders in an organized mammography screening program. Breast Cancer Res Treat. 2001 Nov;70(2):145-53, doi: 10.1023/a:1012939228916.

4. Gilbert FJ, Tucker L, Young KC. Digital breast tomosynthesis (DBT): A review of the evidence for use as a screening tool. Clin Radio. 2015 Dec;71(2):141-50, doi: 10.1016/j.crad.2015.11.008.

5. Vilaverde F, Rocha A, Reis De Sousa M, Mesquita R, Reis A, Mamária T, et al. Tomossíntese mamária: o que o radiologista deve saber. Acta Radiol Port. 2016 Dez;28(109):35-41. doi: https://doi.org/10.25748/arp.10439.

6. ICRU. International Commission on Radiation Units and Measurements. Patient Dosimetry for X Rays Used in Medical Imaging. ICRU Report 74, 2005.

7. Paixão L, Oliveira BB, Viloria C, Oliveira MA de, Teixeira MHA, Nogueira M do S. Monte Carlo derivation of filtered tungsten anode X-ray spectra for dose computation in digital mammography. Radiol Bras. 2016 Nov;48(6):363-7, doi: 10.1590/0100-3984.2014.0108.

8. Oliveira BB, Oliveira MA de, Paixão L, Teixeira MHA, Nogueira M do S. Dosimetria e avaliação da qualidade da imagem em um sistema de radiografia direta. Radiol Bras. 2015 Nov;47(6):361-7. doi: org/10.1590/0100-3984.2013.1876.

9. Hendrick RE, Pisano ED, Averbukh A, Moran C, Berns EA, Yaffe MJ, et al. Comparison of acquisition parameters and breast dose in digital mammography and screen-film mammography in the American College of Radiology imaging network digital mammographic imaging screening trial. Am J Roentgenol. 2010 Feb;194(2):362-9, doi: 10.2214/AJR.08.2114.

10. Schubauer-Berigan MK, Frey GD, Baron L, Hoel DG. Mammography dose in relation to body mass index, race, and menopausal status. Radiat Prot Dosimetry. 2002;98(4):425-32, doi: 10.1093/oxfordjournals.rpd.a006733.

11. Nguyen J V., Williams MB, Patrie JT, Harvey JA. Do women with dense breasts have higher radiation dose during screening mammography? Breast J. 2016 Sep;24(1):35-40. doi: 10.1111/tbj.12833.

12. Shin SU, Chang JM, Bae MS, Lee SH, Cho N, Seo M, et al. Comparative evaluation of average glandular dose and breast cancer detection between single-view digital breast tomosynthesis (DBT) plus single-view digital mammography (DM) and two-view DM: correlation with breast thickness and density. Eur Radiol. 2015 Jan;25(1):1-8, doi: 10.1007/s00330-014-3399-z.

13. Mehnati $\mathrm{P}$, Alizadeh $\mathrm{H}$, Hoda H. Relation between mammographic parenchymal patterns and breast cancer risk: Considering BMI, compressed breast thickness and age of women in Tabriz, Iran. Asian Pacific J Cancer Prev. 2016 Jan;17(4):2259-63, doi: 10.7314/apjcp.2016.17.4.2259.

14. Zulfiqar M, Rohazly I, Rahmah M. Do the majority of Malaysian women have dense breasts on mammogram?. Biomed Imaging Interv J. 2011 Apr;7(2):e14. doi: 10.2349/biij.7.2.e14.

15. Machida Y, Saita A, Namba H, Fukuma E. Automated volumetric breast density estimation out of digital breast tomosynthesis data: feasibility study of a new software version. Springerplus. 2016 Jun;5(1):780. doi: 10.1186/s40064-016-2519-4.

16. IBM SPSS Statistics 15. IBM. 2006. Software. Disponível em: http://www01.ibm.com/software/analytics/spss/products/statistics/ (2006).

17. Imaging B, Journal I, Mehnati P, Alizadeh H, Hoda H, Moshina N, et al. Do the majority of Malaysian women have dense breasts on mammogram? Biomed Imaging Interv J. 2011 Apr;129(1):160-4. doi: 10.2349/biij.7.2.e14.

18. Beckett JR, Kotre CJ, Dance DR, Skinner CL, Klein R, Aichinger H, et al. Dosimetric implications of age related glandular changes in screening mammography. Phys Med Biol. 2000 Mar; 45(3):801- 
13. doi: $10.1088 / 0031-9155 / 45 / 3 / 316$.

19. Dance DR, Skinner CL, Young KC, Beckett JR, Kotre CJ. Additional factors fot the estimation of mean glandular breast dose using the UK mammography dosimetry protocol. Phys Med Biol. 2000 Nov;45(11):3225-40. doi: 10.1088/0031-9155/45/11/308.

20. Mandelson MT, Oestreicher N, Porter PL, White D, Finder CA, Taplin SH, et al. Breast density as a predictor of mammographic detection: comparison of interval- and screen-detected cancers. Breast Cancer Res. 2000 Jul;12(S3):1081-7. doi:10.1093/jnci/92.13.1081.

21. Zoetelief J, Veldkamp WJH, Thijssen MAO, Jansen JTM. Glandularity and mean glandular dose determined for individual women at four regional breast cancer screening units in The Netherlands. Phys Med Biol. 2006 Apr;51(7):1807-17. doi: 10.1088/0031-9155/51/7/012.

22. Young KC, Ramsdale ML, Bignell F. Review of dosimetric methods for mammography in the UK breast screening programme. Radiation Protection Dosimetry. 1998 Nov;80(1-3):183-186. doi: org/10.1093/oxfordjournals.rpd.a032501.

23. Moshina N, Roman M, Waade GG, Sebuodegard S. Breast compression parameters and mammographic density in the Norwegian breast cancer screening programme. Eur Radiol. 2018 Apr;28(40):1662-72. doi: 10.1007/s00330-017-5104-5.

24. Dance DR. Monte-Carlo calculation of conversion factors for the estimation of mean glandular breast dose. Phys Med Biol. 1990 Sep;35(9):1211-9. doi:10.1088/0031-9155/35/9/002. 